\title{
Isotropic Curvature and the Ricci Flow
}

\section{Huy T. Nguyen}

Centre for Mathematics and its Applications, Australian National University, ACT 0200 Australia and Max Planck Institute for Gravitational Physics (Albert Einstein Institute), am Mühlenberg 1, D-14476 Golm, Germany

Correspondence to be sent to: huy.nguyen@aei.mpg.de

In this paper, we study the Ricci flow on higher dimensional compact manifolds. We prove that nonnegative isotropic curvature is preserved by the Ricci flow in dimensions greater than or equal to four. In order to do so, we introduce a new technique to prove that curvature functions defined on the orthonormal frame bundle are preserved by the Ricci flow. At a minimum of such a function, we compute the first and second derivatives in the frame bundle. Using an algebraic construction, we can use these expressions to show that the nonlinearity is positive at a minimum. Finally, using the maximum principle, we can show that the Ricci flow preserves the cone of curvature operators with nonnegative isotropic curvature.

\section{Introduction}

It is well known that various positive curvature conditions imply strong topological restrictions on a Riemannian manifold. One famous example is the $1 / 4$ pinching sphere theorem of Klingernberg, Berger and Rauch, which is a simply connected manifold $\mathcal{M}^{n}$ with globally $1 / 4$ pinched sectional curvatures homeomorphic to a sphere. This theorem was proved using delicate comparison theorems on geodesics. Micallef and Moore [11]

Received July 21, 2008; Revised June 8, 2009; Accepted August 21, 2009

Communicated by John Lott

(C) The Author 2009. Published by Oxford University Press. All rights reserved. For permissions, please e-mail: journals.permissions@oxfordjournals.org. 
used analytic methods to prove a topological sphere theorem for pointwise $1 / 4$ pinched manifolds using harmonic map theory from $\mathbb{S}^{2}$ to $\mathcal{M}^{n}$. They introduced a new curvature condition, a positive isotropic curvature that arose from the complexified second variation of energy of maps from surfaces to $\mathcal{M}$. The condition states that an $n$-dimensional Riemannian manifold, $\mathcal{M}^{n}, n \geq 4$, has positive isotropic curvature if for every orthonormal four-frame, $\left\{e_{1}, e_{2}, e_{3}, e_{4}\right\}$, the curvature operator satisfies

$$
R_{1313}+R_{1414}+R_{2323}+R_{2424}>2 R_{1234}
$$

As pointwise 1/4 pinched sectional curvature implies positive isotropic curvature, Micallef-Moore use this more general condition to prove their sphere theorem. For $n=4$, the theorem above is proved by Hamilton [10] using Ricci flow. In fact, a stronger result is shown in this case, a partial classification of manifolds with positive isotropic curvature up to diffeomorphism. This difficult result is proved using the Ricci flow with surgery; see also [4]. The important first step is to show that positive isotropic curvature is preserved by Ricci flow. However, the proof of this statement is special to dimension four as it uses the self-dual/anti-self-dual decomposition of the curvature operator in dimension four.

In this paper, we shall show that nonnegative isotropic curvature is preserved by the Ricci flow in all dimensions. Let $\left(\mathbb{M}^{n}, g_{i j}\right)$ be a compact, connected $n$-dimensional Riemannian manifold without boundary, the Ricci flow is the following well-known geometric evolution equation:

$$
\frac{\partial}{\partial t} g_{i j}=-2 R_{i j},\left.\quad g_{i j}\right|_{t=0}=g_{i j}(0)
$$

The main result of this paper is to show that the curvature cone of nonnegative isotropic curvature is preserved by the Ricci flow. That is, we will prove the following theorem.

Theorem 1.1 (Nonnegative Isotropic Curvature is Preserved by Ricci Flow). Let $\left(\mathcal{M}^{n}, g_{i j}(t)\right), n \geq 4$ be a solution to the Ricci flow with an initial compact, connected Riemannian metric $\left(\mathcal{M}^{n}, g_{i j}(0)\right)$ that has nonnegative isotropic curvature, then $\left(\mathcal{M}^{n}, g_{i j}(t)\right)$ also has nonnegative isotropic curvature.

We would like to mention that the above theorem was independently proved in [3], where it was used to resolve the $1 / 4$ pinching sectional curvature diffeomorphism sphere theorem. The curvature conditions preserved by the Ricci flow often lead to strong restrictions to the underlying manifold. In particular, we have the following results 
of Böhm and Wilking [2], who showed that manifolds with 2-positive curvature are diffeomorphic to space forms. This extended previous results of [8] and [5] in dimension four. We cannot expect such a result for positive isotropic curvature, for example $\mathbb{S}^{3} \times \mathbb{S}^{1}$ has positive isotropic curvature. In fact, it was proved by Micallef and Wang [12] that positive isotropic is stable under the operation of pointwise connected sum. Hence, we have the following conjecture.

Conjecture 1.2 (see [13]). Let $\left(\mathcal{M}^{n}, g_{i j}\right), n \geq 4$ be a compact Riemannian manifold with positive isotropic curvature. Then a finite cover of $\mathcal{M}^{n}$ is diffeomorphic to $\mathbb{S}^{n}, \mathbb{S}^{n-1} \times \mathbb{S}^{1}$ or a connected sum of these. In particular, the fundamental group is virtually free, that is, there exists a free subgroup of finite index in $\pi_{1}(\mathcal{M})$.

We now describe the proof. As mentioned above, the four-dimensional case relies on the self-dual/anti-self-dual decomposition $\mathfrak{s o} 4=\mathfrak{s o}(3) \oplus \mathfrak{s o}(3)$ of the curvature operator which is special to four dimensions. Hence, in order to prove the theorem, we will use an entirely different method that was developed in [1]. We consider the isotropic curvature as a function of the orthonormal frame bundle of the manifold. Since we use the maximum principle, it suffices to compute pointwise. This allows us to take derivatives in the frame bundle, $\{x, t\} \times O(n) \subset \mathcal{O} \mathcal{M}(n)$. At a minimal frame, the first-order derivatives in the frame bundle vanish and the matrix of second derivatives is convex. However, the entries in the matrix of second-order derivatives are linear in curvature, whereas the nonlinearity is quadratic. To overcome this, we introduce an algebraic construction involving the tensor product of the matrix of second-order derivatives. By choosing appropriate vectors, we can use the positivity of this matrix to show that the nonlinearity is nonnegative at a minimal frame. Hence, the ODE associated the nonlinearity of the Ricci flow that preserves the set of nonnegative isotropic curvatures. By applying the maximum principle, the Ricci flow also preserves this set.

\section{Preliminary Results}

For the convenience of the reader, we collect here some facts that we will use later on.

\subsection{Isotropic curvature}

In this section, we will introduce isotropic curvature. Let $\left(\mathcal{M}^{n}, g_{i j}\right), n \geq 4$ be the Riemannian manifold. Its curvature operator is a self-adjoint mapping on the space 
of two forms, $\mathcal{R}: \Lambda^{2} T_{p} \mathcal{M} \rightarrow \Lambda^{2} T_{p} \mathcal{M}$. Let us consider the complexification of the tangent space and space of two forms $T_{p} \mathcal{M} \otimes \mathbb{C}$ and $\Lambda^{2} T_{p} \mathcal{M} \otimes \mathbb{C}$. We may then extend the inner product of $g($,$) in two different ways: first, we can extend as an Hermitian form$ for $\langle Z, W\rangle=g(Z, \bar{W})$, or in a complex form $(Z, W)=g(Z, W)$ where $Z, W \in T_{p} \mathcal{M} \otimes \mathbb{C}$. The complex sectional curvature is then defined for a complex plane $\sigma=\operatorname{span}\{Z, W\} \subset$ $T_{p} \mathcal{M} \otimes \mathbb{C}$, where $\{Z, W\}$ is a unitary two-frame,

$$
\mathbb{K}_{\mathbb{C}}(\sigma)=\langle\mathcal{R}(Z \wedge W), Z \wedge W\rangle
$$

Then we say $Z$ is isotropic if $(Z, Z)=0$ and $\sigma$ is an isotropic two-plane if $\sigma=\operatorname{span}\{Z, W\}$, where $Z, W$ are isotropic and unitary two-frame. A Riemannian manifold $\left(\mathcal{M}^{n}, g_{i j}\right)$ is said to have nonnegative isotropic curvature if the complex sectional curvature on isotropic two planes is nonnegative, that is,

$$
\mathbb{K}_{\mathbb{C}}(Z, W)=\langle\mathcal{R}(Z \wedge W, Z \wedge W\rangle, \quad \text { and }(Z, Z)=(W, W)=0
$$

Consider the decomposition of complex vector into its real and imaginary parts $Z=X+i Y$, then the condition $(Z, Z)=0$ implies $g(X, X)=g(Y, Y)$ and $g(X, Y)=0$. Hence, any isotropic plane may be written as the span of four orthonormal vectors. In particular, $\left(\mathcal{M}^{n}, g_{i j}\right)$ has nonnegative isotropic curvature if for every orthonormal four-frame, the following curvature condition is satisfied:

$$
R_{1313}+R_{1414}+R_{2323}+R_{2424} \geq \pm 2 R_{1234}
$$

\subsection{Evolution equations}

The Ricci flow is the following quasilinear parabolic system:

$$
\frac{\partial}{\partial t} g_{i j}=-2 R_{i j},\left.\quad g_{i j}\right|_{t=0}=g_{i j}(0)
$$

The following evolution equations may be found in [7] and [9]. Note that we automatically use Uhlenbeck's trick (evolving orthonormal frames) to simplify the evolution equation of the full curvature tensor:

$$
\partial_{t} R_{i j k l}=\triangle R_{i j k l}+2\left(B_{i j k l}-B_{i j l k}+B_{i k j l}-B_{i l j k}\right),
$$

where

$$
B_{i j k l}=R_{p i q j} R_{p k q l}
$$




\section{Refined Maximum Principle}

To prove that the positive isotropic curvature is preserved by the Ricci flow, we will use a refined version the advanced maximum principle; [6, Theorem 4.8]. Let us denote by $\Omega_{X}$, the space of algebraic curvature operators at $X$, that is the set of symmetric bilinear forms $R$ on the space of antisymmetric $(0,2)$-tensors that satisfy the Bianchi identity. We will consider the isotropic curvature function defined on the frame bundle, $\mathcal{O M}=\left\{\left(x, e_{1}, \ldots, e_{n}\right): x \in \mathbb{M}, e_{i} \in T_{x} \mathcal{M}, g_{x}\left(e_{i}, e_{j}\right)=\delta_{i j}\right\}$ by

$$
\begin{aligned}
\mathbb{K}\left(x, e_{1}, \ldots, e_{n}\right)= & R_{x}\left(e_{1}, e_{3}, e_{1}, e_{3}\right)+R_{x}\left(e_{1}, e_{4}, e_{1}, e_{4}\right) \\
& +R_{x}\left(e_{2}, e_{3}, e_{2}, e_{3}\right)+R_{x}\left(e_{2}, e_{4}, e_{2}, e_{4}\right)-2 R_{x}\left(e_{1}, e_{2}, e_{3}, e_{4}\right), \\
= & R_{1313}+R_{1414}+R_{2323}+R_{2424}-2 R_{1234} .
\end{aligned}
$$

Observe that the space of curvature operators with $\mathbb{K}=\{\mathbb{K} \geq 0\} \subset \Omega_{X}$ is a convex set for each $x \in \mathbb{M}$ and invariant under parallel translation. Hence, by the advanced tensor maximum principle, the Ricci flow preserves the condition $\mathbb{K} \geq 0$, if the reaction ODE does. Hence, we will consider any curvature operator in $\Omega_{X}$ such that $\mathbb{K} \geq 0$ for all frames in $\mathcal{O}_{x}$ and suppose that there exists some frame $\left\{e_{1}, \ldots, e_{n}\right\}$ such that $\mathbb{K}=0$. We will consider the first- and second-order conditions for minimality within $\mathcal{O}_{x}$ to deduce inequalities for curvature components. This, in turn, will allow us to show that at a minimum the reaction ODE points into $\mathbb{K}$. We compute the first and second derivatives of $\mathbb{K}$ along the curves in $\mathcal{O}_{x}$, defined by

$$
\frac{d}{d s} e_{i}(s)=\Lambda_{i j} e_{j}(s) ; \quad e_{i}(0)=e_{i}
$$

where $\Lambda$ is an arbitrary antisymmetric 2-tensor. This gives us the following first- and second-order derivatives:

$$
\begin{aligned}
\frac{1}{2} \nabla \mathbb{K}(P)= & \sum_{i, j} \frac{\partial \mathbb{K}}{\partial \Lambda_{i j}} \Lambda_{i j}=\partial_{s} \frac{1}{2} \mathbb{K}(P)=R_{j 313} \Lambda_{1 j}+R_{1 j 13} \Lambda_{3 j}+R_{j 414} \Lambda_{1 j}+R_{1 j 14} \Lambda_{4 j} \\
& +R_{j 323} \Lambda_{2 j}+R_{2 j 23} \Lambda_{3 j}+R_{j 424} \Lambda_{2 j}+R_{2 j 24} \Lambda_{4 j} \\
& -R_{j 234} \Lambda_{1 j}-R_{1 j 34} \Lambda_{2 j}-R_{12 j 4} \Lambda_{3 j}-R_{123 j} \Lambda_{4 j} \\
= & \left(R_{j 313}+R_{j 414}-R_{j 234}\right) \Lambda_{1 j}+\left(R_{j 323}+R_{j 424}-R_{1 j 34}\right) \Lambda_{2 j} \\
& +\left(R_{1 j 13}+R_{2 j 23}-R_{12 j 4}\right) \Lambda_{3 j}+\left(R_{1 j 14}+R_{2 j 24}-R_{123 j}\right) \Lambda_{4 j} .
\end{aligned}
$$


The second derivative equation may be written as

$$
\begin{aligned}
\frac{1}{2} \nabla^{2} \mathbb{K}(P)= & \sum_{i j, k l} \frac{\partial^{2} \mathbb{K}}{\partial \Lambda_{i j} \Lambda_{k l}}=\frac{1}{2} \partial_{s}^{2} \mathbb{K}(P)=R_{k 313} \Lambda_{1 j} \Lambda_{j k}+R_{j k 13} \Lambda_{1 j} \Lambda_{3 k} \\
& +R_{j 3 k 3} \Lambda_{1 j} \Lambda_{1 k}+R_{j 31 k} \Lambda_{1 j} \Lambda_{3 k} \\
& +R_{k 414} \Lambda_{1 j} \Lambda_{j k}+R_{j k 14} \Lambda_{1 j} \Lambda_{4 k}+R_{j 4 k 4} \Lambda_{1 j} \Lambda_{1 k}+R_{j 41 k} \Lambda_{1 j} \Lambda_{4 k} \\
& +R_{k 323} \Lambda_{2 j} \Lambda_{j k}+R_{j k 23} \Lambda_{2 j} \Lambda_{3 k}+R_{j 3 k 3} \Lambda_{2 j} \Lambda_{2 k}+R_{j 32 k} \Lambda_{2 j} \Lambda_{3 k} \\
& +R_{k 424} \Lambda_{2 j} \Lambda_{j k}+R_{j k 24} \Lambda_{2 j} \Lambda_{4 k}+R_{j 4 k 4} \Lambda_{2 j} \Lambda_{2 k}+R_{j 42 k} \Lambda_{2 j} \Lambda_{4 k} \\
& +R_{k j 13} \Lambda_{3 j} \Lambda_{1 k}+R_{1 k 13} \Lambda_{3 j} \Lambda_{j k}+R_{1 j k 3} \Lambda_{3 j} \Lambda_{1 k}+R_{1 j 1 k} \Lambda_{3 j} \Lambda_{3 k} \\
& +R_{j k 14} \Lambda_{4 j} \Lambda_{1 k}+R_{1 k 14} \Lambda_{4 j} \Lambda_{j k}+R_{1 j k 4} \Lambda_{4 j} \Lambda_{1 k}+R_{1 j 1 k} \Lambda_{4 j} \Lambda_{4 k} \\
& +R_{j k 23} \Lambda_{3 j} \Lambda_{2 k}+R_{2 k 23} \Lambda_{3 j} \Lambda_{j k}+R_{2 j k 3} \Lambda_{3 j} \Lambda_{2 k}+R_{2 j 2 k} \Lambda_{3 j} \Lambda_{3 k} \\
& +R_{j k 24} \Lambda_{4 j} \Lambda_{2 k}+R_{2 k 24} \Lambda_{4 j} \Lambda_{j k}+R_{2 j k 4} \Lambda_{4 j} \Lambda_{2 k}+R_{2 j 2 k} \Lambda_{4 j} \Lambda_{4 k} \\
& -R_{k 234} \Lambda_{1 j} \Lambda_{j k}-R_{j k 34} \Lambda_{1 j} \Lambda_{2 k}-R_{j 2 k 4} \Lambda_{1 j} \Lambda_{3 k}-R_{j 23 k} \Lambda_{1 j} \Lambda_{4 k} \\
& -R_{k j 34} \Lambda_{2 j} \Lambda_{1 k}-R_{1 k 34} \Lambda_{2 j} \Lambda_{j k}-R_{1 j k 4} \Lambda_{2 j} \Lambda_{3 k}-R_{1 j 3 k} \Lambda_{2 j} \Lambda_{4 k} \\
& -R_{k 2 j 4} \Lambda_{3 j} \Lambda_{1 k}-R_{1 k j 4} \Lambda_{3 j} \Lambda_{2 k}-R_{12 k 4} \Lambda_{3 j} \Lambda_{j k}-R_{12 j k} \Lambda_{3 j} \Lambda_{4 k} \\
& -R_{k 23 j} \Lambda_{4 j} \Lambda_{1 k}-R_{1 k 3 j} \Lambda_{4 j} \Lambda_{2 k}-R_{12 k j} \Lambda_{4 j} \Lambda_{3 k}-R_{123 k} \Lambda_{4 j} \Lambda_{j k}
\end{aligned}
$$

We expand this expression and collect like terms.

Proposition 3.1. Let $\mathbb{K}$ denote the isotropic curvature. Then, at a space frame minimum, the second derivative of $\mathbb{K}$ at $\{x\} \times \mathfrak{s o}(n)$ is

$$
\begin{aligned}
\nabla^{2} \mathbb{K}= & \left(R_{j k 12}+R_{j 21 k}-R_{j 1 k 2}-2 R_{j k 34}\right) \Lambda_{1 j} \Lambda_{2 k}+\left(R_{j k 13}+R_{j 31 k}-R_{j 1 k 3}-2 R_{j 2 k 4}\right) \Lambda_{1 j} \Lambda_{3 k} \\
& +\left(R_{j k 14}+R_{j 41 k}-R_{j 1 k 4}-2 R_{j 23 k}\right) \Lambda_{1 j} \Lambda_{4 k}+\left(R_{j k 23}+R_{j 32 k}-R_{j 2 k 3}-2 R_{1 j k 4}\right) \Lambda_{2 j} \Lambda_{3 k} \\
& +\left(R_{j k 24}+R_{j 42 k}-R_{j 2 k 4}-2 R_{1 j 3 k}\right) \Lambda_{2 j} \Lambda_{4 k}+\left(R_{j k 34}+R_{j 43 k}-R_{j 3 k 4}-2 R_{1 j 2 k}\right) \Lambda_{3 j} \Lambda_{4 k} \\
& +\left(R_{k 313}+R_{k 414}-R_{k 234}\right) \Lambda_{1 j} \Lambda_{j k}+\left(R_{k 323}+R_{k 424}-R_{1 k 34}\right) \Lambda_{2 j} \Lambda_{j k} \\
& +\left(R_{1 k 13}+R_{2 k 23}-R_{12 k 4}\right) \Lambda_{3 j} \Lambda_{j k}+\left(R_{1 k 14}-R_{2 k 24}-R_{123 k}\right) \Lambda_{4 j} \Lambda_{j k} \\
& +\left(R_{j 3 k 3}+R_{j 4 k 4}\right) \Lambda_{1 j} \Lambda_{1 k}+\left(R_{3 j 3 k}+R_{j 4 k 4}\right) \Lambda_{2 j} \Lambda_{2 k} \\
& +\left(R_{1 j 1 k}+R_{2 j 2 k}\right) \Lambda_{3 j} \Lambda_{3 k}+\left(R_{1 j 1 k}+R_{2 j 2 k}\right) \Lambda_{4 j} \Lambda_{4 k} .
\end{aligned}
$$

\section{Four-Dimensional Isotropic Curvature}

In this section, we will show that in dimension four, nonnegative isotropic curvature is preserved by the Ricci flow. This result was first proved in [10]; however, that proof 
relied on the special decomposition of the Riemann curvature tensor in dimension four. The proof below does not require such a structure. Furthermore, the proof will only rely upon first-order derivatives, and this will allow us to use it in the higher dimensional cases.

Theorem 4.1 [10, see Theorem B1.2]. The Ricci flow on a compact four-manifold preserves positive isotropic curvature.

For the sake of convenience, we write the evolution equations for the Ricci flow:

$$
\begin{aligned}
\frac{\partial R_{i j i j}}{\partial t} & =\triangle R_{i j i j}+2\left(B_{i j i j}+B_{i i j j}-2 B_{i j j i}\right) \\
& =\triangle R_{i j i j}+2\left(R_{i p j q}^{2}+R_{i p i q} R_{j p j q}-2 R_{i p j q} R_{j p i q}\right) .
\end{aligned}
$$

The components of the isotropic curvature are as follows:

$$
\begin{aligned}
\frac{d R_{1313}}{d t}= & R_{1313}^{2}+R_{1323}^{2}+R_{1334}^{2}+R_{1213}^{2}-R_{1232}^{2}+R_{1234}^{2}+R_{1413}^{2}+R_{1423}^{2}-R_{1434}^{2} \\
& -4 R_{2143} R_{1432}+R_{1212} R_{3232}+R_{1414} R_{4343}+2 R_{1412} R_{2343}, \\
\frac{d R_{2424}}{d t}= & R_{2424}^{2}+R_{2414}^{2}+R_{2443}^{2}+R_{2124}^{2}-R_{2141}^{2}+R_{2143}^{2}+R_{2324}^{2}+R_{2314}^{2}-R_{2343}^{2} \\
& -4 R_{1234} R_{2341}+R_{2121} R_{4141}+R_{2323} R_{3434}+2 R_{2321} R_{1434} \\
\frac{d R_{1414}}{d t}= & R_{1414}^{2}+R_{1424}^{2}+R_{1443}^{2}+R_{1214}^{2}-R_{1242}^{2}+R_{1243}^{2}+R_{1314}^{2}+R_{1324}^{2}-R_{1343}^{2} \\
& -4 R_{2134} R_{1342}+R_{1212} R_{4242}+R_{1313} R_{3434}+2 R_{1312} R_{2434} \\
\frac{d R_{2323}}{d t}= & R_{2323}^{2}+R_{2313}^{2}+R_{2334}^{2}+R_{2123}^{2}-R_{2131}^{2}+R_{2134}^{2}+R_{2423}^{2}+R_{2413}^{2}-R_{2434}^{2} \\
& -4 R_{1243} R_{2431}+R_{2121} R_{3131}+R_{2424} R_{4343}+2 R_{2421} R_{1343}, \\
\frac{d R_{1234}}{d t}= & B_{1234}-B_{1243}+B_{1324}-B_{1423} .
\end{aligned}
$$

$$
\begin{aligned}
& B_{1234}=R_{1223} R_{3243}+R_{1221} R_{3241}+R_{1423} R_{3443}+R_{1421} R_{3441} \\
& B_{1243}=R_{1224} R_{4234}+R_{1221} R_{4231}+R_{1324} R_{4334}+R_{1321} R_{4331} \\
& B_{1324}=R_{1332} R_{2342}+R_{1331} R_{2341}+R_{1432} R_{2442}+R_{1431} R_{2441} \\
& B_{1423}=R_{1442} R_{2432}+R_{1441} R_{2431}+R_{1342} R_{2332}+R_{1341} R_{2331}
\end{aligned}
$$


Now, after making the obvious cancellations, we have the following evolution equation:

$$
\begin{aligned}
& \frac{d}{d t} \mathbb{K}(P)=\frac{d}{d t}\left(R_{1313}+R_{1414}+R_{2323}+R_{2424}-2 R_{1234}\right), \\
& =R_{1212} \mathbb{K}(P)+R_{3434} \mathbb{K}(P) \\
& \left\{\begin{array}{l}
-2 R_{2323} R_{1342}+R_{1342}^{2}+R_{2323}^{2}-2 R_{1414} R_{1342}+R_{1414}^{2}+R_{1342}^{2} \\
+2 R_{1314} R_{1323}+R_{1314}^{2}+R_{1342}^{2}+2 R_{1442} R_{2432}+R_{1424}^{2}+R_{2423}^{2} \\
+2 R_{2424} R_{1432}+R_{2424}^{2}+R_{1432}^{2}+2 R_{1313} R_{1432}+R_{1313}^{2}+R_{1423}^{2} \\
-2 R_{1431} R_{2441}+R_{1442}^{2}+R_{1413}^{2}-2 R_{1332} R_{2342}+R_{1323}^{2}+R_{2324}^{2}
\end{array}\right. \\
& +2 R_{1312} R_{2434}+2 R_{2421} R_{1343}+2 R_{1224} R_{4234}-2 R_{1321} R_{1334} \text { ， } \\
& +2 R_{1412} R_{2343}+2 R_{2321} R_{1434}-2 R_{1223} R_{3243}-2 R_{1421} R_{3441} \text { ， } \\
& +4 R_{1234}^{2}-4 R_{12334} R_{1342}+4 R_{1234} R_{1432}-4 R_{1243} R_{2431}+4 R_{1234} R_{2341} .
\end{aligned}
$$

We choose a minimal frame so that $\mathbb{K}(P)=0$ or $R_{1313}+R_{1414}+R_{2323}+R_{2424}=$ $2 R_{1234}$. Furthermore, at this minimal frame by (4), we have

$$
\begin{gathered}
R_{1434}-R_{1232}-R_{3234}+R_{1214}=0 \\
R_{1343}-R_{2124}+R_{4243}-R_{1213}=0
\end{gathered}
$$

We note here that we do not require positivity of the matrix, $\nabla^{2} \mathbb{K}$. We obtain the following cross terms. We explicitly compute one such term, the others follow by permutation. Consider the cross terms:

$$
\begin{aligned}
2 & R_{1312} R_{2434}+2 R_{2421} R_{1343}+2 R_{1224} R_{4234}-2 R_{1321} R_{1334} \\
& =2 R_{1242}\left(R_{4243}+R_{1343}\right)+2 R_{1312}\left(R_{2434}+R_{1343}\right) \\
& =2\left(R_{1242}+R_{1312}\right)\left(R_{2434}+R_{1343}\right) \\
& =2\left(R_{2434}+R_{1343}\right)^{2}
\end{aligned}
$$

where the equality on the third line follows by (11). Similarly, we have the equation

$$
2 R_{1412} R_{2343}+2 R_{2321} R_{1434}-2 R_{1223} R_{3243}-2 R_{1421} R_{3441}=2\left(R_{1214}-R_{1232}\right)^{2} .
$$

The terms (7) simplify as squares:

$$
\begin{aligned}
&-2 R_{1332} R_{2342}+R_{1323}^{2}+R_{2324}^{2}=\left(R_{1323}-R_{2324}\right)^{2}, \\
&-2 R_{1431} R_{2441}+R_{1442}^{2}+R_{1413}^{2}=\left(R_{1413}-R_{1424}\right)^{2}, \\
& 2 R_{1442} R_{2432}+R_{1424}^{2}+R_{2423}^{2}=\left(R_{1424}+R_{2423}\right)^{2}, \\
& 2 R_{1314} R_{1323}+R_{1414}^{2}+R_{1342}^{2}=\left(R_{1314}+R_{1323}\right)^{2},
\end{aligned}
$$


and as

$$
\begin{aligned}
-2 R_{1414} R_{1342}+R_{1414}^{2}+R_{1342}^{2} & =\left(R_{1414}+R_{1324}\right)^{2}, \\
-2 R_{2323} R_{1342}+R_{1342}^{2}+R_{2323}^{2} & =\left(R_{2323}+R_{1324}\right)^{2}, \\
2 R_{1313} R_{1432}+R_{1313}^{2}+R_{1423}^{2} & =\left(R_{1313}-R_{1423}\right)^{2}, \\
2 R_{2424} R_{1432}+R_{2424}^{2}+R_{1432}^{2} & =\left(R_{2424}-R_{1423}\right)^{2} .
\end{aligned}
$$

We may use the Bianchi identity, $R_{1423}=R_{1324}+R_{1234}$, in the last four terms to get

$$
\begin{aligned}
\left(R_{1414}+R_{1324}\right)^{2}+ & \left(R_{2323}+R_{1324}\right)^{2}+\left(R_{1313}+R_{1423}\right)^{2}+\left(R_{2424}+R_{1423}\right)^{2} \\
= & \left(R_{1414}+R_{1423}\right)^{2}+\left(R_{2323}+R_{1423}\right)^{2}+\left(R_{1313}+R_{1324}\right)^{2}+\left(R_{2424}+R_{1324}\right)^{2} \\
& -2 R_{1234}\left(R_{1313}+R_{1414}+R_{2323}+R_{2424}-2 R_{1234}\right)+4 R_{1234}^{2} \\
= & \left(R_{1414}+R_{1423}\right)^{2}+\left(R_{2323}+R_{1423}\right)^{2}+\left(R_{1313}+R_{1324}\right)^{2}+\left(R_{2424}+R_{1324}\right)^{2} \\
& -2 R_{1234} \mathbb{K}(P)+4 R_{1234}^{2} .
\end{aligned}
$$

And the fully mixed terms are dealt with as follows:

$$
4 R_{1234}^{2}-8 R_{1234} R_{1324}-8 R_{1234} R_{1432}=4 R_{1234}\left(R_{1234}-2 R_{1324}-2 R_{1432}\right)=-4 R_{1234}^{2} \text { ， }
$$

where we used the Bianchi identity in the second line, $R_{1432}+R_{1324}=R_{1234}$. Hence, the evolution equation summing (13)-(17) and the term $\left(R_{1212}+R_{2323}\right) \mathbb{K}(P)$ (the term $R_{1234}^{2}$ cancels precisely), we get the following evolution equation:

$$
\begin{aligned}
\frac{d}{d t} \mathbb{K}(P)= & \frac{d}{d t}\left(R_{1313}+R_{1414}+R_{2323}+R_{2424}-2 R_{1234}\right), \\
= & \left(R_{1212}+R_{3434}-2 R_{1234}\right) \mathbb{K}(P)+\left(R_{2323}-R_{1432}\right)^{2}+\left(R_{1414}-R_{1432}\right)^{2} \\
& +\left(R_{2424}-R_{1324}\right)^{2}+\left(R_{1313}-R_{1324}\right)^{2}+\left(R_{1314}+R_{1323}\right)^{2}+\left(R_{1424}+R_{2423}\right)^{2} \\
& +\left(R_{1413}-R_{1424}\right)^{2}+\left(R_{1323}-R_{2324}\right)^{2}+2\left(R_{1214}-R_{1232}\right)^{2}+2\left(R_{2434}+R_{1343}\right)^{2} .
\end{aligned}
$$

As $\mathbb{K}(P)=0$ implies that

$$
R_{1212} \mathbb{K}(P)+R_{3434} \mathbb{K}(P)-2 R_{1234} \mathbb{K}(P)=0,
$$

we have the inequality

$$
\frac{d}{d t} \mathbb{K} \geq 0
$$

when we are at a minimal frame. By the maximum principle, this proves Theorem 4.1. 
Remark 4.2. We remark here that the above computation only made use of the two facts: first, at a zero minimum, we have $\mathbb{K}(P)=0$ and second that $\nabla \mathbb{K}(P)=0$. We did not require the fact that $\nabla^{2} \mathbb{K}$ is positive semidefinite.

Corollary 4.3. Let $g_{i j}$ be a solution to the Ricci flow and suppose we have a space-time frame $\left\{x, e_{1}, \ldots, e_{4}\right\}$ such that,

$$
R_{1313}+R_{1414}+R_{2323}+R_{2424}-2 R_{1234}=\mathbb{K}(P)=0
$$

and $\nabla \mathbb{K}(P)=0$, that is

$$
\begin{aligned}
& R_{1434}-R_{1232}-R_{3234}+R_{1214}=0 \\
& R_{1343}-R_{2124}+R_{4243}-R_{1213}=0
\end{aligned}
$$

then $\frac{d}{d t} \mathbb{K}(P) \geq 0$.

\section{Five-Dimensional Isotropic Curvature}

In this section, we prove that Ricci flow preserves positive isotropic curvature for $n=5$. This dimension differs from higher dimensions as there are no mixed terms, that is, curvature terms that contain two vectors not lying on the minimal isotropic plane.

Theorem 5.1. Let $\left(\mathcal{M}^{5}, g_{i j}(t)\right)$ be a solution to the Ricci flow on $[0, T)$ such that the initial metric $\left(\mathcal{M}^{5}, g_{i j}(0)\right)$ has nonnegative isotropic curvature. Then $\left(\mathcal{M}^{5}, g_{i j}(t)\right)$ has nonnegative isotropic curvature.

\subsection{First-order equalities and second-order inequalities}

Let $\mathbb{K}(P)$ denote the isotropic curvature of an isotropic plane $P$. At a space-time frame maximum, we have the following equalities:

$$
\nabla \mathbb{K}=\sum_{i, j} \frac{\partial \mathbb{K}}{\partial \Lambda_{i j}} \Lambda_{i j}=0
$$


In the component form

$$
\begin{aligned}
& \frac{1}{2} \frac{\partial \mathbb{K}}{\partial \Lambda_{12}}=\frac{1}{2} \frac{\partial \mathbb{K}}{\partial \Lambda_{34}}=0 \\
& \frac{1}{2} \frac{\partial \mathbb{K}}{\partial \Lambda_{13}}=\frac{1}{2} \frac{\partial \mathbb{K}}{\partial \Lambda_{24}}=R_{2313}+R_{2414}-R_{1323}-R_{1424}=0 \\
& \frac{1}{2} \frac{\partial \mathbb{K}}{\partial \Lambda_{14}}=\frac{1}{2} \frac{\partial \mathbb{K}}{\partial \Lambda_{23}}=-R_{1213}+R_{3424}-R_{1334}+R_{1224}=0 \\
& \frac{1}{2} \frac{\partial \mathbb{K}}{\partial \Lambda_{15}}=R_{1353}+R_{5414}-R_{5234}=0 \\
& \frac{1}{2} \frac{\partial \mathbb{K}}{\partial \Lambda_{25}}=R_{5323}+R_{5424}-R_{1534}=0 \\
& \frac{1}{2} \frac{\partial \mathbb{K}}{\partial \Lambda_{35}}=R_{1315}+R_{2523}-R_{1254}=0 \\
& \frac{1}{2} \frac{\partial \mathbb{K}}{\partial \Lambda_{45}}=R_{1514}+R_{2524}-R_{1235}=0 .
\end{aligned}
$$

Furthermore, the following matrix is positive semidefinite:

$$
\nabla^{2} \mathbb{K}=\sum_{i, j, k, l} \frac{\partial^{2} \mathbb{K}}{\partial \Lambda_{i j} \partial \Lambda_{k l}} \geq 0
$$

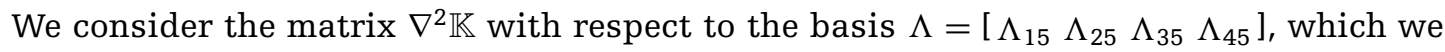
will denote by $M$ :

$$
M=\left[\begin{array}{cccc}
-R_{1313}-R_{1414} & & & \\
+R_{3535}+R_{4545} & -R_{1323}-R_{1424} & -R_{1535}-R_{2545} & -R_{1545}+R_{2535} \\
+R_{1234} & & -R_{1434}+R_{2343} & -R_{1343}-R_{2434} \\
& -R_{2323}-R_{2424} & -R_{3525}+R_{1545} & -R_{2545}-R_{1535} \\
-R_{1323}-R_{1424} & +R_{3535}+R_{4545} & -R_{2434}-R_{1343} & -R_{3423}+R_{1434} \\
& +R_{1234} & & \\
& & -R_{1313}-R_{2323} & \\
-R_{1535}-R_{2545} & -R_{3525}+R_{1545} & +R_{1515}+R_{2525} & -R_{1314}-R_{2423} \\
-R_{1434}+R_{2343} & -R_{2434}-R_{1343} & +R_{1234} & \\
& & & -R_{1414}-R_{2424} \\
-R_{1545}+R_{2535} & -R_{2545}-R_{1535} & -R_{1314}-R_{2423} & +R_{1515}+R_{2525} \\
-R_{1343}-R_{2434} & -R_{3423}+R_{1434} & & +R_{1234}
\end{array}\right] .
$$


Let

$$
\begin{array}{ll}
A=-R_{1535}-R_{2545}, & B=-R_{1434}+R_{2343} \\
C=-R_{1545}+R_{2535}, & D=-R_{1343}-R_{2434} \\
E=-R_{1323}-R_{1424}, & F=-R_{1314}-R_{2423} .
\end{array}
$$

The above matrix may be written succinctly as

$$
M=\left[\begin{array}{cccc}
-R_{1313}-R_{1414} & & & \\
+R_{3535}+R_{4545} & E & A+B & \\
+R_{1234} & & & \\
& -R_{2323}-R_{2424} & & \\
E & +R_{3535}+R_{4545} & -C+D & A-B \\
& +R_{1234} & & \\
& & -R_{1313}-R_{2323} & \\
A+B & & +R_{1515}+R_{2525} & F \\
& & +R_{1234} & -R_{1414}-R_{2424} \\
& & & +R_{1515}+R_{2525} \\
& A-B & & +R_{1234}
\end{array}\right] .
$$

Note that we will often use the shorthand $\mathbb{M}_{i j k l}=\mathbb{M}\left(\Lambda_{i j}, \Lambda_{k l}\right)$ to represent entries of the second derivative matrix.

\subsection{Evolution equation}

We now turn to the evolution equation. First, we note that the five-dimensional isotropic curvature evolution equation contains the four-dimensional isotropic evolution equation, which by Proposition 4.3 is positive at a minimal frame. We will denote this by $\frac{\partial}{\partial t} \mathbb{K}_{4}$ and will only concern ourselves with terms involving the frame $e_{5}$. The remaining curvature terms have a very intriguing structure; they may firstly be separated into two types: the first type contains curvatures that appear in the first derivatives and the second type contains curvatures that only appear in the second derivative. The first type may be further refined: one set has one first derivative term multiplied by the curvature term that appears nowhere in the first or the second derivative (27). They are automatically zero at a minimal frame. The second set has curvature terms that appear in a first derivative (28)(31), which turn out after some manipulation to be nonnegative at a minimum. The second type (26), which contains only second derivatives, is crucial to the entire argument. It 
contains good positive terms that may be written as a linear combination of diagonal entries of the second derivative matrix and bad negative terms that are the off-diagonal terms of the second derivative matrix. To control these terms, we must, of course, use the semipositivity of the matrix. Note that the second derivative matrix has terms that are linear in curvature whereas the nonlinearity is quadratic. We will overcome this later by introducing a generalized determinant.

$$
\begin{aligned}
& \frac{\partial}{\partial t} \mathbb{K}=\frac{\partial}{\partial t} \mathbb{K}_{4}+\left(R_{1515}+R_{2525}\right)\left(R_{3535}+R_{4545}\right)-R_{1535}^{2}-R_{1545}^{2}-R_{2535}^{2}-R_{2545}^{2} \\
& -2 R_{1535} R_{2545}+2 R_{1545} R_{2535} \text {, } \\
& {\left[\begin{array}{l}
+2 R_{1215}\left(R_{3532}+R_{2454}+R_{3541}-R_{4531}\right)+2 R_{1252}\left(R_{1454}+R_{1353}+R_{3245}-R_{4235}\right) \\
+2 R_{3435}\left(R_{1514}+R_{2425}+R_{1523}-R_{1325}\right)+2 R_{3454}\left(R_{1315}+R_{2325}+R_{1425}-R_{1524}\right)
\end{array}\right],} \\
& +2 R_{2534}^{2}+R_{2453}^{2}+R_{2354}^{2}-4 R_{2435} R_{3425}-4 R_{5234} R_{2345}+R_{3153}^{2}+R_{4154}^{2} \\
& -2 R_{1335} R_{2345}+2 R_{1445} R_{2435} \text {, } \\
& +2 R_{5143}^{2}+R_{4153}^{2}+R_{3154}^{2}-4 R_{4153} R_{4351}-4 R_{3154} R_{3451}+R_{3253}^{2}+R_{4254}^{2} \\
& -2 R_{1435} R_{2445}+2 R_{1345} R_{2335} \\
& +2 R_{1253}^{2}+R_{5123}^{2}+R_{5213}^{2}-4 R_{2153} R_{2351}-4 R_{5213} R_{5312}+R_{5224}^{2}+R_{5114}^{2} \\
& -2 R_{1532} R_{2542}+2 R_{1541} R_{2531} \text {, } \\
& +2 R_{1254}^{2}+R_{5124}^{2}+R_{5214}^{2}-4 R_{1254} R_{1542}-4 R_{1254} R_{1452}+R_{1315}^{2}+R_{2325}^{2} \\
& -2 R_{1531} R_{2541}+2 R_{1542} R_{2532} \text {. }
\end{aligned}
$$

We may rewrite the equation in terms of the first- and second-order quantities for $\mathbb{K}$ at a minimal frame.

Claim 5.2. At a minimal space-time frame, the evolution equation above may be rewritten as

$$
\begin{gathered}
\frac{\partial}{\partial t} \mathbb{K}_{5}=\frac{\partial}{\partial t} \mathbb{K}_{4}+\frac{1}{4}\left(\mathbb{M}_{1515}+\mathbb{M}_{2525}\right)\left(\mathbb{M}_{3535}+\mathbb{M}_{4545}\right)-A^{2}-C^{2}, \\
+R_{1215} \frac{\partial \mathbb{K}}{\partial \Lambda_{25}}+R_{1252} \frac{\partial \mathbb{K}}{\partial \Lambda_{15}}+R_{3435} \frac{\partial \mathbb{K}}{\partial \Lambda_{45}}+R_{3454} \frac{\partial \mathbb{K}}{\partial \Lambda_{35}}, \\
+\left(R_{1353}+R_{2435}\right)^{2}+\left(R_{1454}+R_{2354}\right)^{2}+\left(R_{2454}+R_{1345}\right)^{2}+\left(R_{2353}+R_{1435}\right)^{2}, \\
+\left(R_{2524}+R_{1352}\right)^{2}+\left(R_{1415}+R_{1532}\right)^{2}+\left(R_{1513}+R_{1542}\right)^{2}+\left(R_{2523}+R_{1452}\right)^{2} .
\end{gathered}
$$


Proof. Assuming that the isotropic curvature is zero, the term (26) follows easily,

$$
\begin{aligned}
\mathbb{M}_{1515}+\mathbb{M}_{2525}= & -R_{1313}-R_{2323}+R_{1515}+R_{2525}+R_{1234}-R_{1414}-R_{2424}+R_{1515} \\
& +R_{2525}+R_{1234}=2\left(R_{1515}+R_{2525}\right), \\
\mathbb{M}_{3535}+\mathbb{M}_{4545}= & -R_{2323}-R_{2424}+R_{3535}+R_{4545}+R_{1234}-R_{1313}-R_{1414}+R_{3535} \\
& +R_{4545}+R_{1234}=2\left(R_{3535}+R_{4545}\right),
\end{aligned}
$$

and

$$
\begin{aligned}
A^{2}+C^{2} & =\left(R_{1535}+R_{2545}\right)^{2}+\left(-R_{1545}+R_{2535}\right)^{2} \\
& =R_{1535}^{2}+R_{1545}^{2}+R_{2535}^{2}+R_{2545}^{2}+2 R_{1535} R_{2545}-2 R_{1545} R_{2535}
\end{aligned}
$$

This gives us term (32). Next, we will simplify term (28):

$$
\begin{aligned}
& 2 R_{2534}^{2}+R_{2453}^{2}+R_{2354}^{2}-4 R_{2534}\left(R_{2435}-R_{2345}\right) \\
& \quad+R_{3153}^{2}+R_{4154}^{2}+\left[-2 R_{1335} R_{2345}+2 R_{1445} R_{2435}\right] .
\end{aligned}
$$

Applying the Bianchi identity, $R_{2345}+R_{2453}+R_{2534}=0$, to the term in the brackets, we get

$$
4 R_{2534}\left(R_{2345}-R_{2435}\right)=-4 R_{2534}^{2}
$$

which allows us to simplify (36):

$$
2 R_{2534}^{2}+R_{2453}^{2}+R_{2354}^{2}-4 R_{2435} R_{3425}-4 R_{5234} R_{2345}=R_{2453}^{2}+R_{2354}^{2}-2 R_{2534}^{2} \text {. }
$$

Again, using the Bianchi identity, $R_{2345}=R_{2435}-R_{2534}$, on the terms in (37), we get

$$
\begin{aligned}
2 R_{1353} R_{2345}-2 R_{1454} R_{2435} & =2 R_{1353}\left(R_{2435}-R_{2534}\right)-2 R_{1454}\left(R_{2345}+R_{2534}\right) \\
& =2 R_{1353} R_{2435}-2 R_{1454} R_{2345}-2 R_{2534}\left(R_{1353}+R_{1454}\right) \\
& =2 R_{1353} R_{2435}-2 R_{1454} R_{2345}+2 R_{2534}^{2}
\end{aligned}
$$

where the last line follows from

$$
\frac{1}{2} \frac{\partial \mathbb{K}}{\partial \Lambda_{15}}=R_{1353}+R_{1454}-R_{5234}=0 \Longrightarrow R_{1353}+R_{1454}=-R_{2534} .
$$

Hence,

$$
\begin{aligned}
& 2 R_{2534}^{2}+R_{2453}^{2}+R_{2354}^{2}-4 R_{2534}\left(R_{2435}-R_{2345}\right)+R_{3153}^{2}+R_{4154}^{2}-2 R_{1335} R_{2345}+2 R_{1445} R_{2435} \\
& \quad=R_{2453}^{2}+R_{2354}^{2}-2 R_{2534}^{2}+2 R_{1353} R_{2435}-2 R_{1454} R_{2345}+2 R_{2534}^{2}+R_{1353}^{2}+R_{1454}^{2} \\
& \quad=\left(R_{1353}+R_{2435}\right)^{2}+\left(R_{1454}-R_{2345}\right)^{2} .
\end{aligned}
$$


Terms (29)-(31) simplify by a similar argument,

$$
\begin{aligned}
& 2 R_{1254}^{2}+R_{5124}^{2}+R_{5214}^{2}-4 R_{1254}\left(R_{1542}+R_{1452}\right)+R_{1315}^{2}+R_{2325}^{2}-2 R_{1531} R_{2541}+2 R_{1542} R_{2532} \\
& \quad=\left(R_{1513}+R_{1542}\right)^{2}+\left(R_{2523}-R_{1452}\right)^{2} \\
& 2 R_{1253}^{2}+R_{5123}^{2}+R_{5213}^{2}-4 R_{1253}\left(R_{5213}-R_{2351}\right)+R_{5224}^{2}+R_{5114}^{2}-2 R_{1532} R_{2542}+2 R_{1541} R_{2531} \\
& \quad=\left(R_{2524}+R_{1352}\right)^{2}+\left(R_{1415}-R_{1532}\right)^{2}
\end{aligned}
$$

and

$$
\begin{aligned}
& 2 R_{5143}^{2}+R_{4153}^{2}+R_{3154}^{2}-4 R_{4351}\left(R_{4153}-R_{3154}\right)+R_{3253}^{2}+R_{4254}^{2}-2 R_{1435} R_{2445}+2 R_{1345} R_{2335} \\
& \quad=\left(R_{2454}+R_{1345}\right)^{2}+\left(R_{2353}-R_{1435}\right)^{2} .
\end{aligned}
$$

This completes the proof of the claim.

By the advanced maximum principle, to prove that $\mathbb{K}_{5}$ is preserved by the Ricci flow, it suffices to prove the following claim.

Claim 5.3. At a zero space-frame minimum, $\left\{x, e_{1}, \ldots, e_{5}\right\}$, we have the following inequality:

$$
\frac{1}{4}\left(\mathbb{M}_{1515}+\mathbb{M}_{2525}\right)\left(\mathbb{M}_{3535}+\mathbb{M}_{4545}\right) \geq-A^{2}+C^{2}
$$

In order to prove this claim, we will introduce a method to extract quadratic estimates from the semipositivity of the matrix $M$. First note that if a matrix $\mathbb{M}: V \rightarrow V$ is semipositive, then the tensor product $\mathbb{M} \otimes \mathbb{M}$ is positive semidefinite. Next, consider the wedge product $v \wedge w$, which belongs to the space $V \otimes V$,

$$
v \wedge w=\frac{1}{\sqrt{2}}(v \otimes w-w \otimes v)
$$

Then we have

$$
\mathbb{M} \otimes \mathbb{M}(v \wedge w, v \wedge w)=\mathbb{M}(v, v) \mathbb{M}(w, w)-\mathbb{M}(v, w)^{2} \geq 0
$$

which is the determinant of a two-by-two submatrix. However, we can use sums of wedge products of vectors to obtain more sophisticated estimates, which is what we will do in the following. We now apply this to our positive semidefinite matrix $\mathbb{M}$ above with $V=\mathfrak{s o}(5)$ and vectors $v=\Lambda_{i j}$. 
Proof. Here, we will exploit the fact that $\mathbb{M}$ is positive semidefinite. We apply the following vectors:

$$
\begin{aligned}
& V_{1}^{-}=\left(\Lambda_{15} \wedge \Lambda_{35}-\Lambda_{25} \wedge \Lambda_{45}\right) \\
& V_{2}^{+}=\left(\Lambda_{15} \wedge \Lambda_{45}-\Lambda_{25} \wedge \Lambda_{35}\right)
\end{aligned}
$$

to the positive semidefinite matrix $M \otimes M$, and we get the following inequalities:

$\mathbb{M} \otimes \mathbb{M}\left(V_{1}^{-}, V_{1}^{-}\right)$,

$$
\begin{aligned}
\mathbb{M}_{1515} \mathbb{M}_{3535}+\mathbb{M}_{2525} \mathbb{M}_{4545} & \geq(A+B)^{2}+(A-B)^{2}-2(C+D)(-C+D)+2 E F \\
& =2 A^{2}+2 B^{2}-2\left(D^{2}-C^{2}\right)+2 E F
\end{aligned}
$$

$\mathbb{M} \otimes \mathbb{M}\left(V_{2}^{+}, V_{2}^{+}\right)$,

$$
\begin{aligned}
\mathbb{M}_{1515} \mathbb{M}_{4545}+\mathbb{M}_{2525} \mathbb{M}_{3535} & \geq(C+D)^{2}+(-C+D)^{2}+2(A+B)(A-B)-2 E F \\
& =2 C^{2}+2 D^{2}+2\left(A^{2}-B^{2}\right)-2 E F .
\end{aligned}
$$

Adding (39) to (40),

$$
\left(\mathbb{M}_{1515}+\mathbb{M}_{2525}\right)\left(\mathbb{M}_{3535}+\mathbb{M}_{4545}\right) \geq 4 A^{2}+4 C^{2}
$$

Proof (Proof of Theorem 5.1). By Claims 5.2 and 5.3, we see that at a minimal space frame, we have

$$
\frac{d}{d t} \mathbb{K} \geq 0
$$

By the refined maximum principle, this shows that $\mathbb{K}_{5}$ is preserved by the Ricci flow.

\section{Higher Dimensional Isotropic Curvature}

In this section, we consider nonnegative isotropic curvature in dimensions six and higher. Here, the nonlinearity contains curvature terms with two components that lie off the minimal isotropic plane.

Theorem 6.1. Let $\left(\mathcal{M}^{n}, g_{i j}(t)\right)$ be a Ricci flow with initial nonnegative isotropic curvature. Then $\left(\mathcal{M}^{n}, g_{i j}(t)\right)$ also has nonnegative isotropic curvature. 


\subsection{First- and second-order quantities}

At a critical point, we have $\nabla \mathbb{K}=0$. This gives us the following identities, $5 \leq k \leq n$ :

$$
\begin{aligned}
& \frac{1}{2} \frac{\partial \mathbb{K}}{\partial \Lambda_{12}}=\frac{1}{2} \frac{\partial \mathbb{K}}{\partial \Lambda_{34}}=0 \\
& \frac{1}{2} \frac{\partial \mathbb{K}}{\partial \Lambda_{13}}=\frac{1}{2} \frac{\partial \mathbb{K}}{\partial \Lambda_{24}}=R_{2313}+R_{2414}-R_{1323}-R_{1424}=0 \\
& \frac{1}{2} \frac{\partial \mathbb{K}}{\partial \Lambda_{14}}=\frac{1}{2} \frac{\partial \mathbb{K}}{\partial \Lambda_{23}}=-R_{1213}+R_{3424}-R_{1334}+R_{1224}=0 \\
& \frac{1}{2} \frac{\partial \mathbb{K}}{\partial \Lambda_{1 k}}=R_{13 k 3}+R_{k 414}-R_{k 234}=0 \\
& \frac{1}{2} \frac{\partial \mathbb{K}}{\partial \Lambda_{2 k}}=R_{k 323}+R_{k 424}-R_{1 k 34}=0 \\
& \frac{1}{2} \frac{\partial \mathbb{K}}{\partial \Lambda_{3 k}}=R_{131 k}+R_{2 k 23}-R_{12 k 4}=0 \\
& \frac{1}{2} \frac{\partial \mathbb{K}}{\partial \Lambda_{4 k}}=R_{1 k 14}+R_{2 k 24}-R_{123 k}=0 .
\end{aligned}
$$

At a minimal point, we have $\nabla^{2} \mathbb{K} \geq 0$. We consider the matrix $M$ where $\nabla^{2}=$ $\Lambda^{t} M \Lambda$, where $\Lambda$ is the basis

$$
\left[\Lambda_{12} \Lambda_{13} \Lambda_{14} \Lambda_{23} \Lambda_{24} \sum_{k=5}^{n} \Lambda_{1 k} \Lambda_{2 k} \Lambda_{3 k} \Lambda_{4 k} \sum_{k \neq l, k>l} \Lambda_{k l}\right]
$$

We will only require the part of matrix corresponding to

$$
\left[\begin{array}{llllllll}
\Lambda_{1 k} & \Lambda_{2 k} & \Lambda_{3 k} & \Lambda_{4 k} & \Lambda_{1 l} & \Lambda_{2 l} & \Lambda_{3 l} & \Lambda_{4 l}
\end{array}\right]
$$

This has the following form:

$$
\mathbb{M}=\left[\begin{array}{ll}
M_{k} & N_{k l} \\
N_{k l} & M_{l}
\end{array}\right]
$$


where the individual matrices $M_{k}, M_{l}, N_{k l}$ have the explicit form:

$$
M_{k}=\left[\begin{array}{cccc}
-R_{1313}-R_{1414} & & -R_{1 k 3 k}-R_{2 k 4 k} & -R_{1 k 4 k}+R_{2 k 3 k} \\
+R_{3 k 3 k}+R_{4 k 4 k} & -R_{1323}-R_{1424} & -R_{1434}+R_{2343} & -R_{1343}-R_{2434} \\
+R_{1234} & & & \\
-R_{1323}-R_{1424} & +R_{3 k 3 k}+R_{4 k 4 k} & -R_{3 k 2 k}+R_{1 k 4 k} & -R_{2 k 4 k}-R_{1 k 3 k} \\
& +R_{1234} & -R_{2434}-R_{1343} & -R_{3423}+R_{1434} \\
-R_{1 k 3 k}-R_{2 k 4 k} & -R_{3 k 2 k}+R_{1 k 4 k} & -R_{1313}-R_{2323} & \\
-R_{1434}+R_{2343} & -R_{2434}-R_{1343} & +R_{1 k 1 k}+R_{2 k 2 k} & -R_{1314}-R_{2423} \\
& & +R_{1234} & \\
-R_{1 k 4 k}+R_{2 k 3 k} & -R_{2 k 4 k}-R_{1 k 3 k} & -R_{1314}-R_{2423} & +R_{1 k 1 k}+R_{2 k 2 k} \\
-R_{1343}-R_{2434} & -R_{3423}+R_{1434} & & +R_{1234}
\end{array}\right] \text {, }
$$

and

$$
N_{k l}=\left[\begin{array}{cccc}
R_{3 k 3 l}+R_{4 k 4 l} & -R_{k l 34} & R_{13 k l}+R_{1 l k 3} & R_{14 k l}+R_{1 l k 4} \\
& & -R_{k 2 l 4} & -R_{k 23 l} \\
-R_{l k 34} & R_{3 k 3 l}+R_{4 k 4 l} & R_{k l 23}+R_{k 32 l} & R_{24 k l}+R_{2 l k 4} \\
R_{1 k l 3}+R_{13 l k} & R_{l k 23}+R_{2 k l 3} & -R_{1 l k 4} & -R_{1 k 3 l} \\
-R_{l 2 k 4} & -R_{1 l k 4} & R_{1 l 1 k}+R_{2 k 2 l} & -R_{12 k l} \\
R_{l k 14}+R_{1 k l 4} & R_{l k 24}+R_{2 k l 4} & -R_{12 l k} & R_{1 k 1 l}+R_{2 k 2 l} \\
-R_{l 23 k} & -R_{1 l 3 k} & &
\end{array}\right]
$$

Using the following expressions:

$$
\begin{array}{cl}
A_{k}=-R_{1 k 3 k}-R_{2 k 4 k}, & B=-R_{1434}+R_{2343}, \\
C_{k}=-R_{1 k 4 k}+R_{2 k 3 k}, & D=-R_{1343}-R_{2434}, \\
E=-R_{1323}-R_{1424}, & F=-R_{1314}-R_{2423}
\end{array}
$$


the first matrix may be rewritten as follows:

$$
M_{k}=\left[\begin{array}{cccc}
-R_{1313}-R_{1414} & & & \\
+R_{3 k 3 k}+R_{4 k 4 k} & E & A_{k}+B & C_{k}+D \\
+R_{1234} & & & \\
& -R_{2323}-R_{2424} & & \\
E & +R_{3 k 3 k}+R_{4 k 4 k} & -C_{k}+D & A_{k}-B \\
& +R_{1234} & & \\
& & -R_{1313}-R_{2323} & \\
A_{k}+B & -C_{k}+D & +R_{1 k 1 k}+R_{2 k 2 k} & F \\
& & +R_{1234} & \\
& & F & -R_{1414}-R_{2424} \\
C_{k}+D & A_{k}-B & & +R_{1 k 1 k}+R_{2 k 2 k} \\
& & & +R_{1234}
\end{array}\right]
$$

\subsection{Evolution equation}

The evolution equation for the high-dimensional Ricci flow of isotropic curvature is given by the following formula. As in the five-dimensional case, we group the terms of the evolution equation according to whether they appear in the first or the second derivative. For the second group of terms, we make a further subdivision. First, note that we only have in any curvature term at most two vectors $e_{k}, e_{l}$ that appear off the minimal frame. Group all the terms that appear with only one vector. These terms will appear only in the matrix $\mathbb{M}_{k}$ and may be handled like in the five-dimensional case. The remaining terms will contain curvature with two nonminimal vectors. They are precisely the vectors that appear in the matrix $N_{k l}$. Using this, grouping the following evolution equation is a straightforward but long computation:

$$
\begin{gathered}
\frac{\partial}{\partial t} \mathbb{K}_{k}=\frac{\partial}{\partial t} \mathbb{K}_{4}, \\
\sum_{k=5}^{n}\left[\frac{1}{4}\left(\mathbb{M}_{1 k 1 k}+\mathbb{M}_{2 k 2 k}\right)\left(\mathbb{M}_{3 k 3 k}+\mathbb{M}_{4 k 4 k}\right)-A_{k}^{2}-C_{k}^{2}\right], \\
+\sum_{k=5}^{n}\left[+R_{121 k} \frac{\partial \mathbb{K}}{\partial \Lambda_{2 k}}+R_{343 k} \frac{\partial \mathbb{K}}{\partial \Lambda_{4 k}}+R_{34 k 4} \frac{\partial \mathbb{K}}{\partial \Lambda_{4 k}}+R_{34 k 4} \frac{\partial \mathbb{K}}{\partial \Lambda_{3 k}}\right],
\end{gathered}
$$




$$
\begin{gathered}
+\sum_{k=5}^{n}\left[\begin{array}{c}
\left(R_{13 k 3}+R_{243 k}\right)^{2}+\left(R_{14 k 4}-R_{23 k 4}\right)^{2} \\
+\left(R_{24 k 4}+R_{134 k}\right)^{2}+\left(R_{23 k 3}-R_{143 k}\right)^{2} \\
+\left(R_{2 k 24}+R_{13 k 2}\right)^{2}+\left(R_{141 k}-R_{1 k 32}\right)^{2} \\
+\left(R_{1 k 13}+R_{1 k 42}\right)^{2}+\left(R_{2 k 23}-R_{14 k 2}\right)^{2}
\end{array}\right], \\
\sum_{k, l=5, k \neq l}^{n}\left[\left(R_{k 1 l 3}-R_{l 1 k 3}\right)^{2}+\left(R_{k 2 l 4}-R_{l 2 k 4}\right)^{2}+\left(R_{l 1 k 4}-R_{k 1 l 4}\right)^{2}+\left(R_{l 2 k 3}-R_{k 2 l 3}\right)^{2}\right], \\
-2 \sum_{k, l=5, k \neq l}^{n}\left[\left(R_{1 k 3 l}+R_{2 l 4 k}\right)\left(R_{1 l 3 k}+R_{2 k 4 l}\right)+\left(R_{1 l 4 k}-R_{2 k 3 l}\right)\left(R_{1 k 4 l}-R_{2 l 3 k}\right)\right], \\
+2 \sum_{k, l=5, k \neq l}^{n}\left[R_{12 k l} R_{k l 34}-\left(R_{1 k 1 l}+R_{2 k 2 l}\right)\left(R_{3 k 2 l}+R_{4 k 4 l}\right)\right], \\
\left.\left.\sum_{k, l=5, k \neq l}^{n} R_{1 k 3 l} R_{2 l 4 k}\right)\left(R_{1 l 3 k}+R_{2 k 4 l}\right)+\left(R_{1 l 4 k}-R_{2 k 3 l}\right)\left(R_{1 k 4 l}-R_{2 l 3 k}\right)\right] .
\end{gathered}
$$

\subsection{Proof of Theorem 6.1}

To prove the main theorem, we use the same technique as in dimension five, but now we must account for the cross terms. Fortunately, the vectors used in that proof work for the higher dimensional case and the cross terms are precisely accounted for.

Proof. By applying the vectors

$$
\begin{aligned}
& V_{1}^{-}=\sum_{k=5}^{n}\left[\Lambda_{1 k} \wedge \Lambda_{3 k}-\Lambda_{2 k} \wedge \Lambda_{4 k}\right], \\
& V_{2}^{+}=\sum_{k=5}^{n}\left[\Lambda_{1 k} \wedge \Lambda_{4 k}+\Lambda_{2 k} \wedge \Lambda_{3 k}\right],
\end{aligned}
$$

to the positive semidefinite matrix $\mathbb{M} \otimes \mathbb{M}$, we get the following inequalities:

$\mathbb{M} \otimes \mathbb{M}\left(V_{1}^{-}, V_{1}^{-}\right):$

$$
\begin{aligned}
& \sum_{k=5}^{n}\left[\mathbb{M}_{1 k 1 k} \mathbb{M}_{3 k 3 k}+\mathbb{M}_{2 k 2 k} \mathbb{M}_{4 k 4 k}\right] \geq \sum_{k=5}^{n}\left[\mathbb{M}_{1 k 3 k}^{2}+\mathbb{M}_{2 k 4 k}^{2}-2 \mathbb{M}_{1 k 4 k} \mathbb{M}_{3 k 2 k}-2 \mathbb{M}_{1 k 2 k} \mathbb{M}_{3 k 4 k}\right] \\
& \quad+2 \sum_{k, l=5, k \neq l}^{n}\left[\mathbb{M}_{1 k 3 l} \mathbb{M}_{3 k 1 l}-\mathbb{M}_{1 k 1 l} \mathbb{M}_{3 k 3 l}\right]-2 \sum_{k, l=5, k \neq l}^{n}\left[\mathbb{M}_{1 k 4 l} \mathbb{M}_{3 k 2 l}-\mathbb{M}_{1 k 2 l} \mathbb{M}_{3 k 4 l}\right] \\
& \quad-2 \sum_{k, l=5, k \neq l}^{n}\left[\mathbb{M}_{2 k 3 l} \mathbb{M}_{4 k 1 l}-\mathbb{M}_{2 k 1 l} \mathbb{M}_{4 k 3 k}\right]+2 \sum_{k, l=5, k \neq l}^{n}\left[\mathbb{M}_{2 k 4 l} \mathbb{M}_{4 k 2 l}-\mathbb{M}_{2 k 2 l} \mathbb{M}_{4 k 4 l}\right]
\end{aligned}
$$


$\mathbb{M} \otimes \mathbb{M}\left(V_{2}^{+}, V_{2}^{+}\right):$

$$
\begin{aligned}
& \sum_{k=5}^{n}\left[\mathbb{M}_{1 k 1 k} \mathbb{M}_{4 k 4 k}+\mathbb{M}_{2 k 2 k} \mathbb{M}_{3 k 3 k}\right] \geq \sum_{k=5}^{n}\left[\mathbb{M}_{1 k 4 k}^{2}+\mathbb{M}_{2 k 3 k}^{2}+2 \mathbb{M}_{1 k 3 k} \mathbb{M}_{4 k 2 k}-2 \mathbb{M}_{1 k 2 k} \mathbb{M}_{4 k 3 k}\right] \\
& \quad+2 \sum_{k, l=5, k \neq l}^{n}\left[\mathbb{M}_{1 k 4 l} \mathbb{M}_{4 k 1 l}-\mathbb{M}_{1 k 1 l} \mathbb{M}_{4 k 4 l}\right]+2 \sum_{k, l=5, k \neq l}^{n}\left[\mathbb{M}_{1 k 3 l} \mathbb{M}_{4 k 2 l}-\mathbb{M}_{1 k 2 l} \mathbb{M}_{4 k 3 l}\right] \\
& \quad+2 \sum_{k, l=5, k \neq l}^{n}\left[\mathbb{M}_{2 k 4 l} \mathbb{M}_{3 k 1 l}-\mathbb{M}_{2 k 1 l} \mathbb{M}_{3 k 4 l}\right]+2 \sum_{k, l=5, k \neq l}^{n}\left[\mathbb{M}_{2 k 3 l} \mathbb{M}_{3 k 2 l}-\mathbb{M}_{2 k 2 l} \mathbb{M}_{3 k 3 l}\right]
\end{aligned}
$$

Consider the sum of (51) and (52), the first terms on the left-hand side and the right-hand side of the inequality sum to

$$
\begin{aligned}
\left(\mathbb{M}_{1 k 1 k}+\right. & \left.\mathbb{M}_{2 k 2 k}\right)\left(\mathbb{M}_{3 k 3 k}+\mathbb{M}_{4 k 4 k}\right) \geq \mathbb{M}_{1 k 3 k}^{2}+\mathbb{M}_{2 k 4 k}^{2}-2 \mathbb{M}_{1 k 4 k} \mathbb{M}_{3 k 2 k}-2 \mathbb{M}_{1 k 2 k} \mathbb{M}_{3 k 4 k} \\
& +\mathbb{M}_{1 k 4 k}^{2}+\mathbb{M}_{2 k 3 k}^{2}+2 \mathbb{M}_{1 k 3 k} \mathbb{M}_{4 k 2 k}-2 \mathbb{M}_{1 k 2 k} \mathbb{M}_{4 k 3 k} \\
= & 4 A_{k}^{2}+4 C_{k}^{2}
\end{aligned}
$$

The remaining terms simplify as follows:

$$
\begin{aligned}
& -2 \mathbb{M}_{1 k 2 l} \mathbb{M}_{4 k 3 l}+2 \mathbb{M}_{2 k 1 l} \mathbb{M}_{4 k 3 l}+2 \mathbb{M}_{1 k 2 l} \mathbb{M}_{3 k 4 l}-2 \mathbb{M}_{2 k 1 l} \mathbb{M}_{3 k 4 l} \\
& =2\left(\mathbb{M}_{1 k 2 l}-\mathbb{M}_{1 l 2 k}\right)\left(\mathbb{M}_{3 k 4 l}-\mathbb{M}_{4 k 3 l}\right) \\
& =8 R_{12 l k} R_{l k 34},-2 \mathbb{M}_{1 k l l} \mathbb{M}_{4 k 4 l}-2 \mathbb{M}_{2 k 2 k} \mathbb{M}_{3 k 3 l}-2 \mathbb{M}_{1 k 1 l} \mathbb{M}_{3 k 3 l}-2 \mathbb{M}_{2 k 2 l} \mathbb{M}_{4 k 4 l} \\
& =-8\left(R_{1 k 1 l}+R_{2 k 2 l}\right)\left(R_{3 k 3 l}+R_{4 k 4 l}\right), \\
& \mathbb{M}_{1 k 3 l} \mathbb{M}_{4 k 2 l}+\mathbb{M}_{1 k 3 l} \mathbb{M}_{3 k 1 l}+\mathbb{M}_{1 k 4 l} \mathbb{M}_{4 k 1 l}-\mathbb{M}_{1 k 4 l} \mathbb{M}_{3 k 2 l} \\
& \quad+\mathbb{M}_{2 k 4 l} \mathbb{M}_{1 l 3 k}+\mathbb{M}_{2 k 4 l} \mathbb{M}_{4 k 2 l}+\mathbb{M}_{2 k 3 l} \mathbb{M}_{3 k 2 l}-\mathbb{M}_{2 k 3 l} \mathbb{M}_{4 k 1 l} \\
& =\left(\mathbb{M}_{1 l 3 k}+\mathbb{M}_{4 k 2 l}\right)\left(\mathbb{M}_{1 k 3 l}+\mathbb{M}_{2 k 4 l}\right)+\left(\mathbb{M}_{3 k 2 l}-\mathbb{M}_{1 l 4 k}\right)\left(\mathbb{M}_{2 k 3 k}-\mathbb{M}_{1 k 3 l}\right)
\end{aligned}
$$

Using the Bianchi identity, we see that

$$
\mathbb{M}_{1 l 3 k}+\mathbb{M}_{4 k 2 l}=R_{1 k l 3}-R_{13 l k}-R_{l 2 k 4}+R_{l k 24}+R_{2 k l 4}-R_{1 l 3 k}=2 R_{1 l k 3}+2 R_{2 k l 4}
$$

Repeating for the $\mathbb{M}_{1 k 3 l}+\mathbb{M}_{2 k 4 l}$ term, we get the equality

$$
\left(\mathbb{M}_{1 l 3 k}+\mathbb{M}_{4 k 2 l}\right)\left(\mathbb{M}_{1 k 3 l}+\mathbb{M}_{2 k 4 l}\right)=4\left(R_{1 k l 3}+R_{2 k l 4}\right)\left(R_{1 l k 3}+R_{2 l k 4}\right)
$$


After combining these various inequalities, we have

$$
\begin{aligned}
\sum_{k=5}^{n}\left[\frac{1}{4}\left(\mathbb{M}_{1 k 1 k}+\mathbb{M}_{2 k 2 k}\right)\left(\mathbb{M}_{3 k 3 k}+\mathbb{M}_{4 k 4 k}\right)-A_{k}^{2}-C_{k}^{2}\right] \\
\geq+2 \sum_{k, l=5, k \neq l}^{n}\left[\left(R_{1 k 3 l}+R_{2 l 4 k}\right)\left(R_{1 l 3 k}+R_{2 k 4 l}\right)+\left(R_{1 l 4 k}-R_{2 k 3 l}\right)\left(R_{1 k 4 l}-R_{2 l 3 k}\right)\right] \\
\quad-2 \sum_{k, l=5, k \neq l}^{n}\left[R_{12 k l} R_{k l 34}-\left(R_{1 k 1 l}+R_{2 k 2 l}\right)\left(R_{3 k 2 l}+R_{4 k 4 l}\right)\right] \\
\quad+2 \sum_{k, l=5, k \neq l}^{n}\left[\left(R_{1 k 3 l} R_{2 l 4 k}\right)\left(R_{1 l 3 k}+R_{2 k 4 l}\right)+\left(R_{1 l 4 k}-R_{2 k 3 l}\right)\left(R_{1 k 4 l}-R_{2 l 3 k}\right)\right] .
\end{aligned}
$$

Hence, (54) shows that the sum of (44), (48), (49), and (50) is nonnegative. As the remaining terms are strictly positive, at a space-time frame minimum, the following inequality holds:

$$
\frac{d}{d t} \mathbb{K}_{k} \geq 0
$$

Applying the refined maximum principle, we can conclude that $\mathbb{K}_{k}$ is preserved by the Ricci flow.

\section{Acknowledgments}

The author would like to thank his supervisor, Dr. Ben Andrews, for all the help and advice he received during his $\mathrm{PhD}$ candidature. The author would also like to note that this research was partially supported by an Australian Postgraduate Research Award and an ANU supplementary scholarship.

\section{References}

[1] Andrews, B., and H. Nguyen. "Four-manifolds with 1/4-pinched flag curvature." Asian Journal of Mathematics, to appear.

[2] Böhm, C., and B. Wilking. "Manifolds with positive curvature operators are space forms." Annals of Mathematics 167, no. 3 (2008): 1079-97.

[3] Brendle, S., and R. Schoen. "Manifolds with 1/4-pinched curvature are space forms." Journal of the American Mathematical Society 22, no. 1 (2009): 287-307.

[4] Chen, B.-L., and X.-P. Zhu. "Ricci flow with surgery on four-manifolds with positive isotropic curvature." Journal of Differential Geometry 74, no. 2 (2006): 177-264.

[5] Chen, H. "Pointwise 1/4-pinched 4-manifolds." Annals of Global Analysis and Geometry 9, no. 2 (1991): 161-76. 
[6] Chow, B., and D. Knopf. The Ricci Flow: An Introduction. Mathematical Surveys and Monographs 110. Providence, RI: American Mathematical Society, 2004.

[7] Hamilton, R. S. "Three-manifolds with positive Ricci curvature." Journal of Differential Geometry 17, no. 2 (1982): 255-306.

[8] Hamilton, R. S. "Four-manifolds with positive curvature operator." Journal of Differential Geometry 24, no. 2 (1986): 153-79.

[9] Hamilton, R. S. The Formation of Singularities in the Ricci Flow, 7-136. Cambridge, MA: International Press, 1995.

[10] Hamilton, R. S. "Four-manifolds with positive isotropic curvature." Communications in Analysis and Geometry 5, no. 1 (1997): 1-92.

[11] Micallef, M. J., and J. D. Moore. "Minimal two-spheres and the topology of manifolds with positive curvature on totally isotropic two-planes." Annals of Mathematics 127, no. 1 (1988): 199-227.

[12] Micallef, M. J., and M. Y. Wang. "Metrics with nonnegative isotropic curvature." Duke Mathematical Journal 72, no. 3 (1993): 649-72.

[13] Schoen, R. Open problems. Manifolds with Pointwise 1/4-Pinched Curvature. Pacific Northwest Geometry Seminar, University of Oregon, 2007 fall. 